\title{
Recent Progress in the Development of Photomultiplier Tubes
}

\section{Shoei NAKAYAMA*}

Kamioka Observatory, Institute for Cosmic Ray Research, The University of Tokyo

E-mail: shoei@suketto.icrr.u-tokyo.ac.jp

Photomultiplier tubes (PMTs) are extremely sensitive photon detectors, and their history is quite long. PMTs have a very wide application in the high energy and astroparticle physics field. In this document, some of recent activities about the PMT development are described. The HyperKamiokande group has been developing two types of $50 \mathrm{~cm}$ diameter photosensors, both with much better photon detection capability than that of the Super-Kamiokande PMTs. The efforts for applying the innovative technologies to large aperture PMTs are also progressing, such as the developments of the spherical MCP-PMT, LAPPD, VSiPMT, etc. The recent progress of the PMT developments for a collider experiment and dark matter search experiments are also presented briefly.

International Conference on New Photo-detectors PhotoDet2015,

6-9 July 2015

Moscow, Troitsk, Russia

${ }^{*}$ Speaker. 


\section{Introduction}

Photomultiplier tubes (PMTs) are extremely sensitive detectors of light in the visible, ultraviolet (UV), and near infrared ranges. A typical PMT consists of a photocathode, several dynodes, and an anode in a vacuum tube. When photons enter the PMT and strike the photocathode, electrons are emitted as a consequence of the photoelectric effect. These electrons are directed by the focusing electrode toward the electron multiplier, where the number of electrons is multiplied by the process of secondary emission. The electron multiplier is typically made up from a number of metallic electrodes called dynodes.

The history of PMTs is quite long [1]. The first documented demonstration of a PMT was done in 1934. These days SiPM/MPPC have excellent photon detection performance and are evolving rapidly, but there still are many reasons to keep using PMTs, such as low noise, high gain, large photosensitive area, high frequency response, wide dynamic range, etc.

This paper reviews recent research and development activities of vacuum-based photodetectors in the high energy and astroparticle physics fields, mainly those for neutrino experiments. The review is much biased by the author's past and current activities and interests, and omission from this paper does not at all mean less importance.

\section{New large area photodetectors for Hyper-Kamiokande}

One of major applications for vacuum-based photodetectors is water Cherenkov detectors. A ring-imaging Cherenkov detector detects the Cherenkov rings by an array of photodetectors. After photons traveling through several meters of pure water, the spectrum of Cherenkov photons is peaked in the UV range. Since amount of Cherenkov light is very small, high-sensitive photodetectors in the UV and visible ranges are necessary in a water Cherenkov detector. Thus, PMTs can be a strong candidate photodetector.

Hyper-Kamiokande (Hyper-K) is a proposed megaton-scale water Cherenkov detector [2]. Figure 1 shows a schematic view of the Hyper-K detector. In the current baseline design, the detector consists of two $250 \mathrm{~m}$ long water tanks, each containing the two photon sensitive segments separated optically. The inner segment called the Inner Detector (ID) is the main active volume for

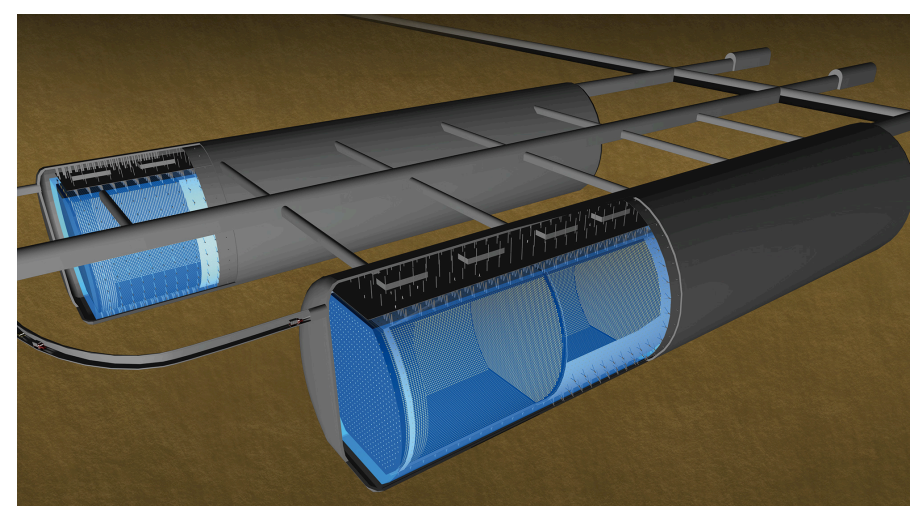

Figure 1: A schematic view of the Hyper-Kamiokande detector. The detector consists of two $250 \mathrm{~m}$ long water tanks. The inner active volume is monitored by a total of $99,00050 \mathrm{~cm}$ diameter photodetectors. 

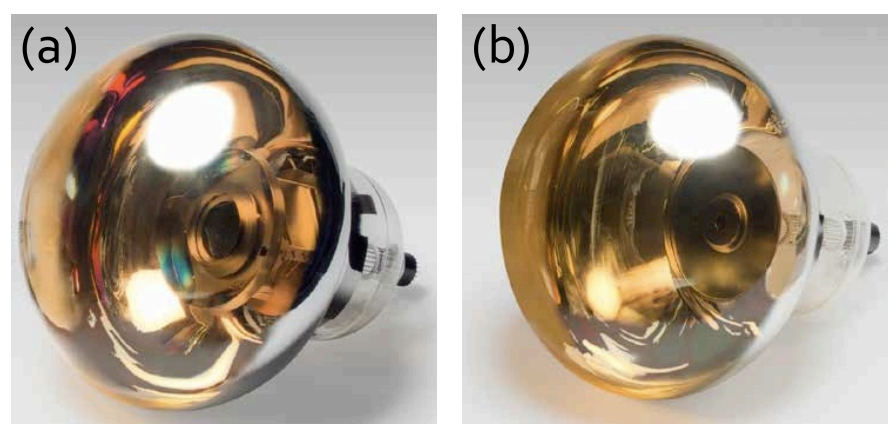

Figure 2: Two types of new 50cm diameter photodetectors for Hyper-K : (a) the PMT using an improved shape of dynodes, and (b) the hybrid photodetector HPD with an avalanche diode as the electron multiplier.

physics measurements, and the outer segments called the Outer Detector (OD) acts mainly as a veto for entering particles such as cosmic ray muons. The total water mass is 0.99 megatons and the fiducial mass is 0.56 megatons, which is 25 times as large as that of the Super-Kamiokande detector. The surface area of the ID for detecting Cherenkov light is about $100,000 \mathrm{~m}^{2}$, requiring huge amount of large area photodetectors. A total of 99,000 photodetectors with a diameter of 50 $\mathrm{cm}$ are used in the ID.

Hamamatsu R3600 $50 \mathrm{~cm}$ PMTs, so-called Super-K PMTs, have an excellent photon detection capability, and their performance stability and low failure rate have been demonstrated by a longterm use (about 20 years) in Super-K. They can be good candidate photodetectors also for Hyper-K. To enhance the detector performance and reduce the cost further, two types of new $50 \mathrm{~cm}$ diameter vacuum-based photodetectors have been developed for Hyper-K. One is a PMT which uses socalled Box-and-Line dynodes, a combination of a box-shaped large acceptance first dynode and linear-focused subsequent dynodes [3, 4]. The other is a hybrid photodetector called HPD, which uses an avalanche diode (AD) as the electron multiplier [5]. Pictures of these new photodetectors for Hyper-K are shown in Figure 2.

The emitted electrons can take various drift paths and sometimes miss a dynode during the electron multiplication in Venetian-Blind type dynodes, such as those in the Super-K PMTs, causing worse timing and charge resolutions. Since the electrons take almost a unique drift path in the Box-and-Line dynodes, the new Hyper-K PMTs, Hamamatsu R12860, have much higher timing and charge resolutions than those of the Super-K PMTs. The timing resolution $\sigma$ is about $1 \mathrm{~ns}$ (2 ns) and the charge resolution is about 35\% (53\%), for the single photon measurement by R12860 (R3600).

The new PMT utilizes also an improved photocathode. As shown in Figure 3 (left), the quantum efficiency of new PMTs reaches $30 \%$ on average at around $400 \mathrm{~nm}$, about 1.4 times higher than that of the Super-K PMT. In addition to the improvement of the photocathode, the photoelectron collection efficiency of the new PMTs is also much higher, benefitted from the large acceptance by the box-shaped first dynode. Combined with the higher "electronics hit" efficiency brought by the higher single photon charge resolution, the total efficiency for the single photon detection of the new Hyper-K PMT is almost twice higher than that of the Super-K PMTs, as shown in Figure 3 (right). Although some demonstration works, such as a long-term performance stability check and 

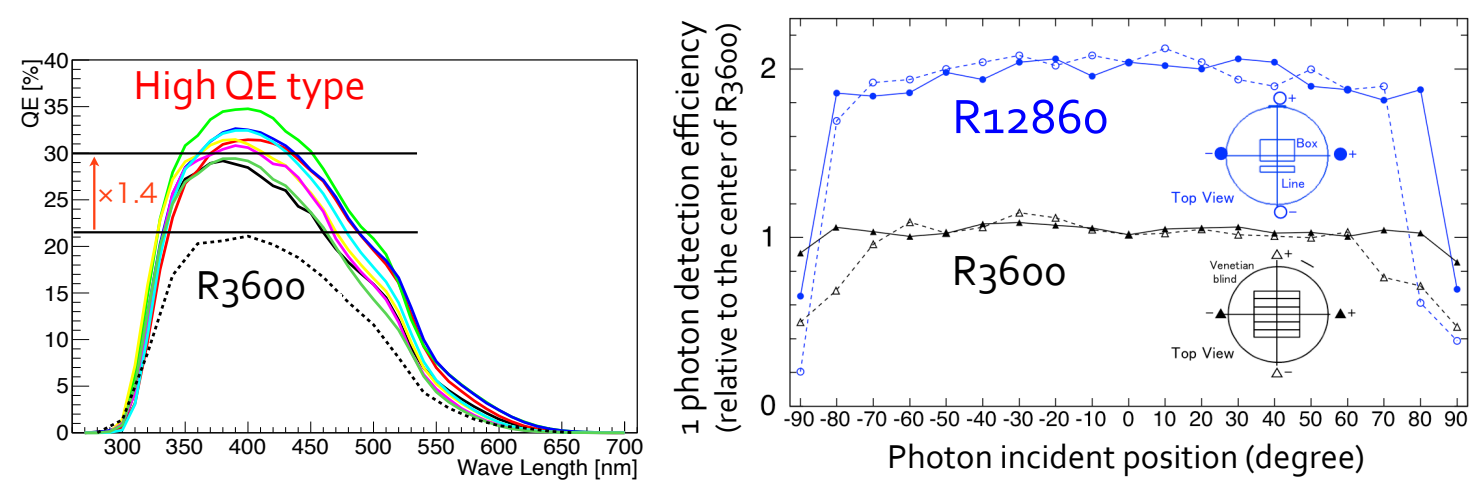

Figure 3: The measured quantum efficiency curves for the high-QE photocathode of new photodetectors for Hyper-K (left) and the single photon detection efficiency of the new Hamamatsu R12860 PMT for Hyper-K as a function of incident positions (right), compared to those for the Super-K PMTs, Hamamatsu R3600.

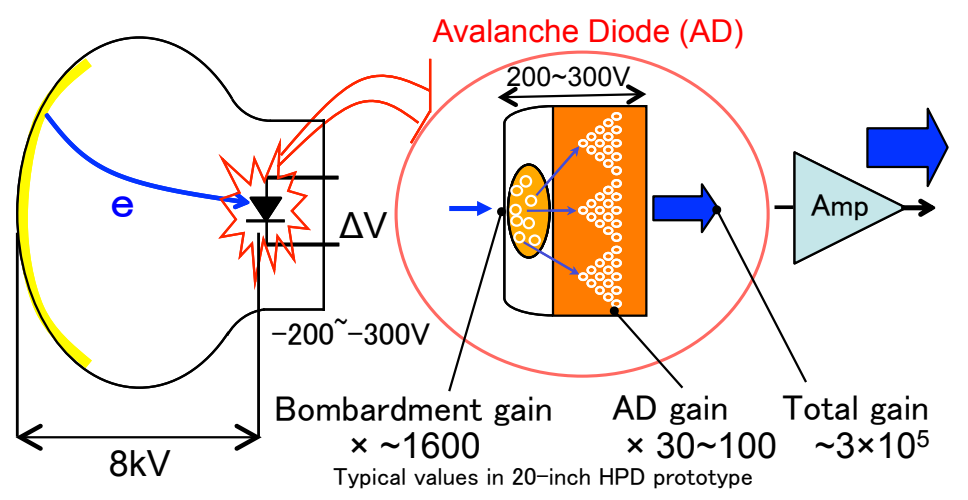

Figure 4: A schematic view of the signal amplification in the new $50 \mathrm{~cm}$ HPD for Hyper-K.

a pressure tolerance check, are still ongoing [6], it can be said that the new PMT, Hamamatsu $\mathrm{R} 12860$, is basically ready for use in Hyper-K.

Figure 4 shows a schematic view of the new HPD for Hyper-K. An AD with a diameter of $20 \mathrm{~mm}$ is used as the electron multiplier in the HPD. To accelerate and focus the emitted photoelectrons into this small $\mathrm{AD}$ area, $8 \mathrm{kV}$ is applied between the photocathode and the AD, which is much higher than the typical supply voltage $1-2 \mathrm{kV}$ for conventional PMTs. This higher operation voltage provides higher bombardment gain, better timing resolution, and less sensitivity to external magnetic field. The gain of the HPD itself is lower than the requirement for Hyper-K, so the HPD is used in combination with a preamplifier.

The prototype $50 \mathrm{~cm}$ HPD which utilizes a smaller $5 \mathrm{~mm}$ AD has shown excellent measurement performance. Especially the charge resolution is far better than that of similarly-sized conventional PMTs, and a charge distribution with separated peaks created by 1-4 photoelectrons can be output as shown in Figure 5. To get sufficient collection efficiency, the final $50 \mathrm{~cm}$ HPD product has to be instrumented with an $\mathrm{AD}$ with a diameter of $20 \mathrm{~mm}$ or lager. However, the development of the $50 \mathrm{~cm}$ HPD with a $20 \mathrm{~mm}$ AD has suffered from poor signal-to-noise ratio caused by the large junction capacitance of the large area AD. As possible solutions to this large noise issue, the use of a segmented $\mathrm{AD}$ and a transformer coupling is under evaluation, along with the development 


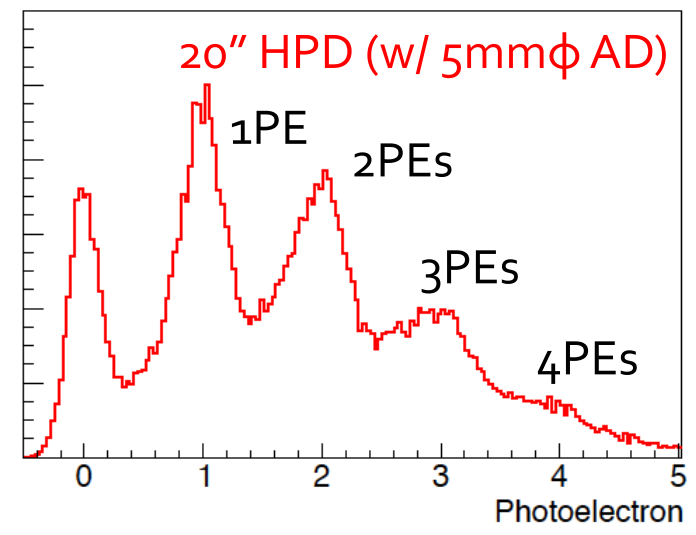

Figure 5: An integrated charge distribution of a prototype $50 \mathrm{~cm}$ HPD which utilizes a $5 \mathrm{~mm}$ diameter AD as the electron multiplication.

of a low noise preamplifier.

More detailed and latest information on the development of the new $50 \mathrm{~cm}$ photodetectors for Hyper-K can be found in other papers within the same conference proceedings $[3,4,5,6]$.

\section{Large area PMT developments in the US and China}

ET Enterprise Limited ADIT is now a US-based PMT manufacturer in Texas. The company has been developing a large area PMT [7]. It's important that such an additional vendor comes in the marketplace for price competition and for supply capacity. Development of an 11-inch PMT with a high quantum efficiency photocathode is supported by NSF, and tests have been performed at the University of Pennsylvania, UC Davis, etc. Figure 6 shows a photograph of a prototype 11inch PMT and the single photoelectron charge distribution obtained by the prototype. The single photoelectron peak is clearly seen with a good peak-to-valley ratio better than 2. If successfully produced, the PMTs may be a cost-effective alternative to Hamamatsu for Hyper-K OD PMTs. They are also candidate PMTs for some US-based projects, such as WATCHMAN.
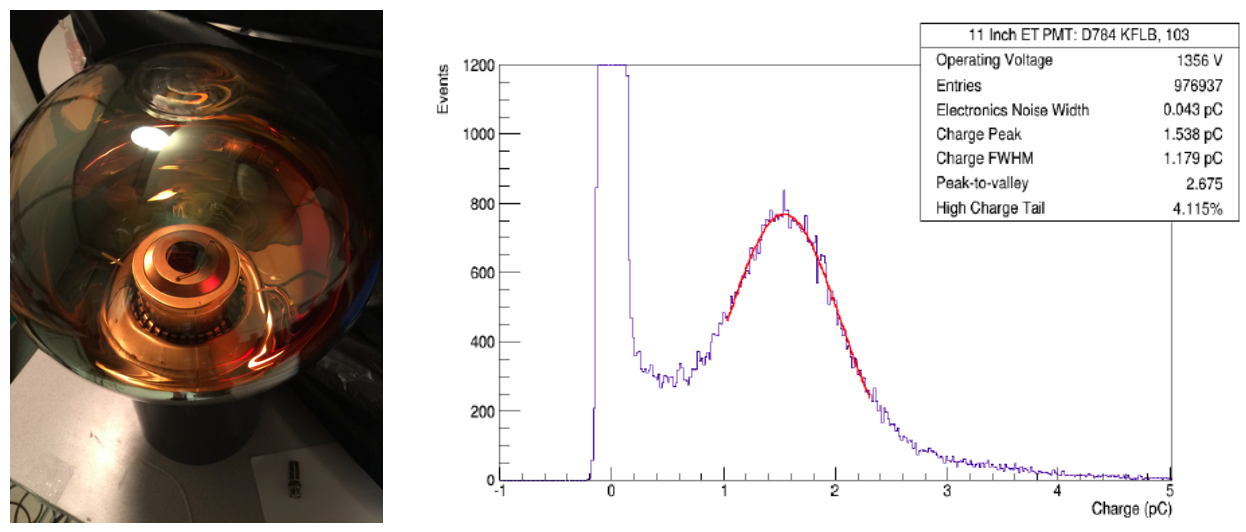

Figure 6: A photograph of the prototype 11-inch high quantum efficiency PMT made by ETEL/ADIT (left) and the single photoelectron charge distribution measured by the prototype PMT (right). 


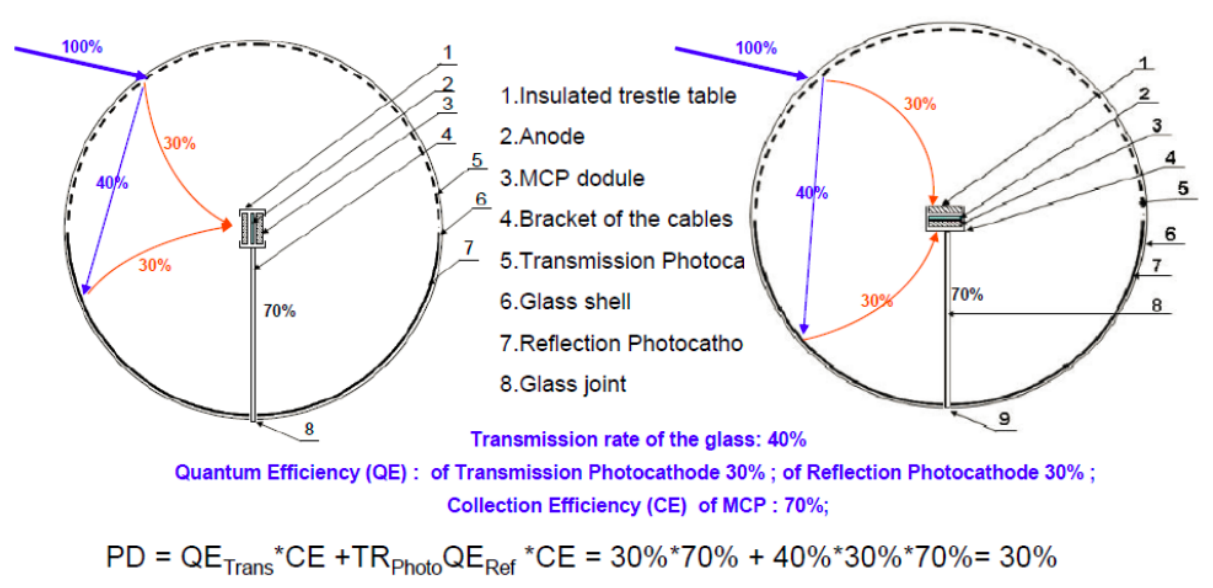

Figure 7: A schematic view of the new MCP-PMTs under development in China for the JUNO experiment. The left (right) one is with the vertical (horizontal) MCPs configuration.

A new type of large area PMT has been under development in China for the JUNO experiment [8], which is a multipurpose neutrino experiment designed primarily to determine the neutrino mass hierarchy by observing reactor neutrinos. A schematic view of the new PMT is shown in Figure 7. The new PMT utilizes a set of two micro-channel plates (MCPs) as the electron multiplier. Aiming to increase the photon detection efficiency, a reflective photocathode is created on the inner surface of the back-side hemisphere of the spherical PMT glass shell with a diameter of $50 \mathrm{~cm}$, in addition to the transmissive photocathode on the front-side hemisphere. Some variations of 20-inch prototypes, such as a spherical PMT with a vertical MCPs configuration and an ellipsoidal PMT with a horizontal MCPs configuration have been produced. Although the basic performance of the initial prototypes is reasonably good, for example the peak-to-valley ratio of the single photoelectron peak higher than 2, new prototypes are under development aiming to increase the quantum efficiency and the photoelectron collection efficiency.

\section{Multi PMT optical module for KM3NeT}

$\mathrm{KM} 3 \mathrm{NeT}$ is a proposed next generation multi- $\mathrm{km}^{3}$ sized neutrino telescope at the bottom of the Mediterranean sea to search for neutrinos from distant astrophysical sources such as supernovae and gamma ray bursts (Figure 8). The faint Cherenkov light emitted by charged particles generated in neutrino-nucleus interactions under deep sea is detected by stand alone sensor modules called Digital Optical Modules, DOMs [9]. A DOM contains 31 of 3-inch PMTs in a 17-inch glass sphere, and thus has some directional sensitivity about an incident photon. The head of each PMT is surrounded by a reflective ring to further increase the light collection efficiency by about $27 \%$. All readout electronics for PMTs and calibration sensors are confined inside the glass sphere of the DOM which has only one penetrator for connection to the backbone cable of the detection unit.

A new generation of 3-inch PMTs has been developed for KM3NeT by several vendors (Figure 9). Two of them, namely ETEL D792 and Hamamatsu R12199, have been satisfactory, providing good measurement performance with low price (per unit photo-cathode area less than 10-inch PMTs). The installation of the detection unit for KM3NeT will start very soon in 2015. 


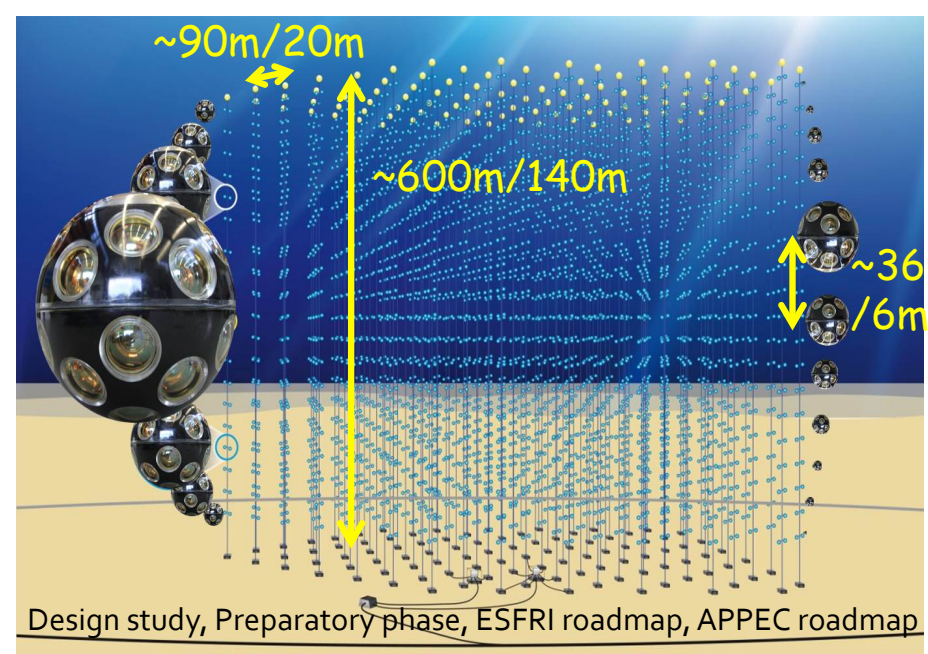

Figure 8: A schematic view of KM3NeT and the multi PMT optical modules.

(a)

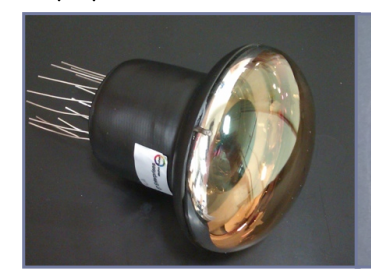

(b)

(c)

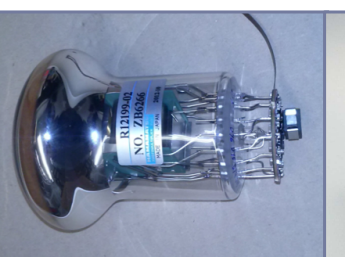

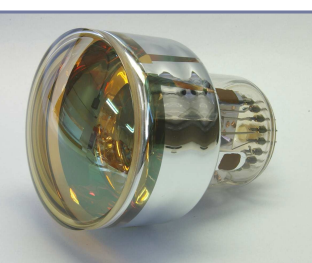

(d)

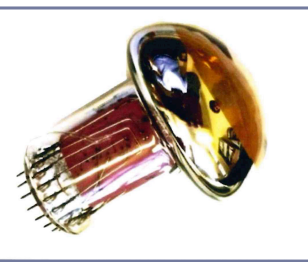

Figure 9: The 3-inch PMTs developed for KM3NeT: (a) ETEL D792, (b) Hamamatsu R12199, (c) HZC XP53B20, and (d) a prototype by MELZ FEU.

\section{Other innovative vacuum-based large area photodetectors}

A group in Naples has been developing an innovative hybrid photodetector called the Vacuum Silicon PhotoMultiplier Tube (VSiPMT) [10]. As one can image from a conceptual drawing shown in Figure 10 (left), the VSiPMT is designed aiming to utilize the excellent characteristics of the SiPM/MPPC technology with a large photo-sensitive window. The VSiPMT is expected to have various attractive features, such as an excellent photon counting capability and fast response, with a very low power consumption thanks to the absence of the standard dynode chain which requires the voltage divider.

To demonstrate the feasibility of the VSiPMT technology, two initial prototypes have been manufactured by Hamamatsu (Figure 10, right), which have a GaAsP photocathode with a diameter of $3 \mathrm{~mm}$ and a special non-windowed MPPC with a dimension of $1 \mathrm{~mm}$ square. A full characterization of the prototypes has been performed in the INFN Napoli laboratory. The results have shown many encouraging performance, especially the unrivaled photon counting capability and nice timing resolution (Transit Time Spread $<0.5 \mathrm{~ns}$ ). The relatively higher dark rate $(>10 \mathrm{kHz}$ @ 0.5 photoelectrons) and smaller dynamic range have to be resolved, and the realization of next VSiPMT prototypes with a larger photocathode area is the next step. 

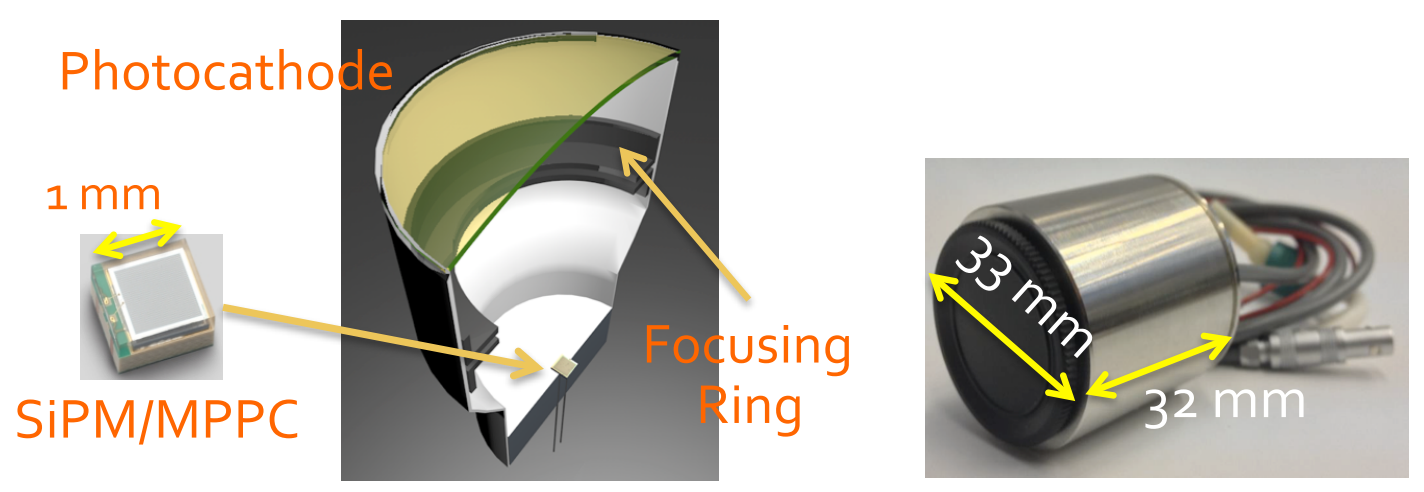

Figure 10: A conceptual design of the VSiPMT and the first prototype by Hamamatsu.
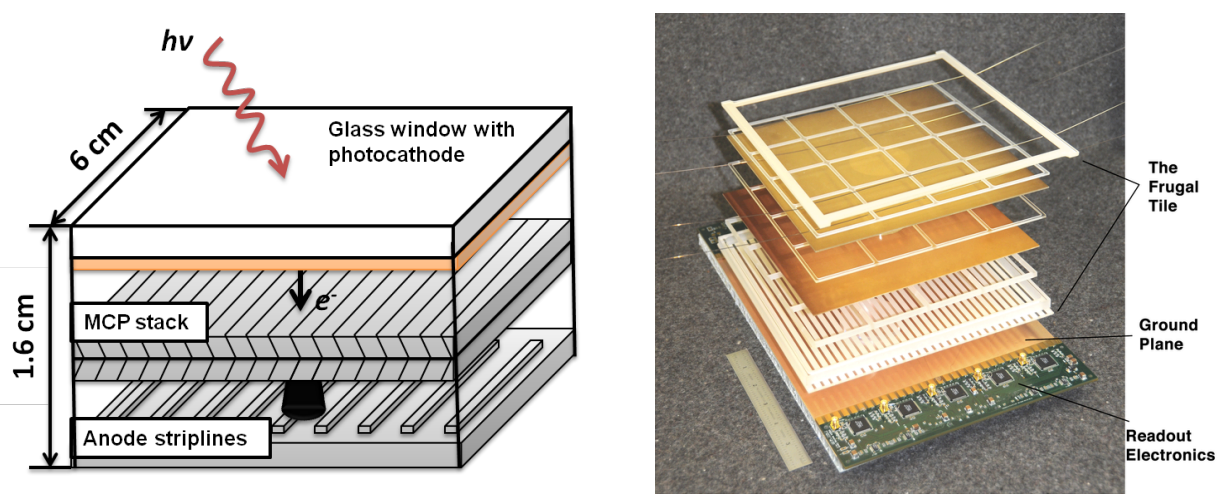

Figure 11: A schematic view of the $6 \mathrm{~cm}$-square prototype LAPPD (left) and composition of the LAPPD glass tile (right).

The Large-Area Picoseconds Photo-Detector (LAPPD) collaboration has been developing a modular photodetector system composed of thin, planar, glass-body modules each with two $20 \mathrm{~cm}$ $\times 20 \mathrm{~cm}$ MCPs [11]. Unlike standard MCP-PMTs which are not designed for large area applications, this large area photodetector system with $<1 \mathrm{~cm}$ spatial resolution and $<100 \mathrm{ps}$ time resolution for the single photon detection would have wide applications such as large water Cherenkov detectors. Figure 11 shows a schematic view of the $6 \mathrm{~cm}$ square prototype LAPPD (left) and composition of the LAPPD glass tile (right). As seen in the figure, LAPPD has rather simple structure, composed of only several parts. As a lower cost and lower risk path towards commercialization of LAPPD, new facility capable of producing $6 \mathrm{~cm}$ square photodetectors has been constructed at Argonne National Laboratory [12].

Photomultipliers have been widely used also in collider experiments. Figure 12 (left) shows a picture of the MCP-PMT developed for the Belle-II TOP (Time Of Propagation) counter [13]. This multi-anode MCP-PMT has a square shape photocathode $(23 \mathrm{~mm} \times 23 \mathrm{~mm})$ to realize a large photocathode coverage. The mass production of more than 500 MCP-PMTs has completed, and the basic performance has been checked. It is found that every PMT has a great Transit Time Spread below 50 psec. Figure 12 (right) shows a picture of the Hybrid Avalanche Photo-Detector (HAPD) developed for the Belle-II Aerogel RICH detector [14]. The HAPD has about $5 \mathrm{~mm}$ position resolution realized by using a 144-channel pixelated APD. As well as the MCP-PMT for 

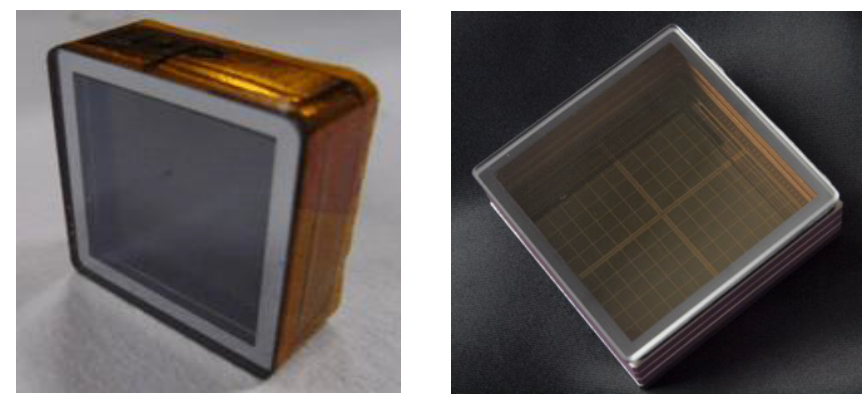

Figure 12: The MCP-PMT for the Belle-II TOP conter (left) and the HAPD for the Belle-II Aerogel RICH (right).
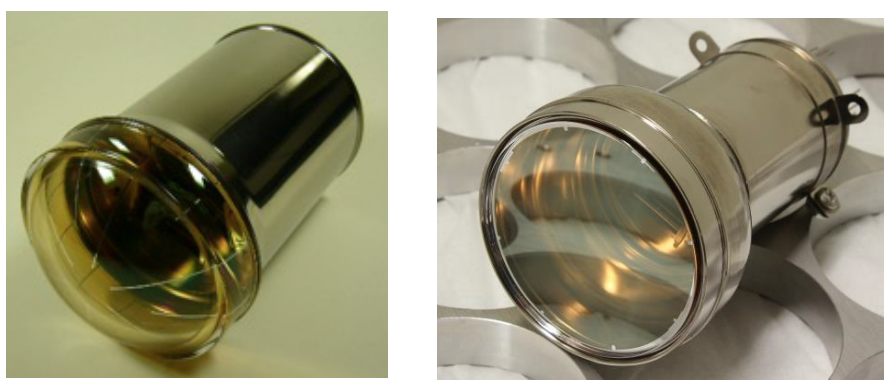

Figure 13: The prototype 3-inch PMT for the XMASS-1.5 dark matter search experiment (left) and the 3-inch PMT for the XENON1T experiment (right).

Belle-II TOP, this HAPD also works well in a $1.5 \mathrm{~T}$ magnetic field. As for the radiation tolerance of the HAPD, no significant QE degradation was observed by a 20 year equivalent aging test. The preparation of more than 400 HAPSs for the Aerogel RICH will be completed soon.

\section{Low radioactive PMT developments for dark matter searches}

The XMASS experiment is aiming to directly detect the cold dark matter [15]. The detector consists of 642 PMTs surrounding the $800 \mathrm{~kg}$ liquid xenon. Since the liquid xenon emits the scintillation light in the vacuum ultraviolet (VUV) region, the PMTs used in the XMASS detector have high sensitivity to the light in this region. The XMASS collaboration has a plan to build the next phase detector, called XMASS-1.5, which will has ten times lager fiducial volume than that of the current XMASS detector. Figure 13 (left) shows a picture of the new PMT (Hamamatsu R13111) under development for the XMASS-1.5 [16]. The new PMT has a fish-eye (or domed) photocathode, which provides a sensitivity to the light from a transverse direction and helps to discriminate the surface background. Since dark matter signals are very rare and most likely also very weak, the intrinsic radiation sources in the detector components have to be eliminated as much as possible. The new PMT for the XMASS-1.5 will have even lower radioactive impurities than those in the current XMASS PMTs. The target radioactive contamination per PMT is below 0.1 $\mathrm{mBq}$ for the U/Th chain, $0.3 \mathrm{mBq}$ for ${ }^{60} \mathrm{Co}$, and $1 \mathrm{mBq}$ for ${ }^{40} \mathrm{~K}$.

Figure 13 (right) shows a picture of new 3-inch PMT (Hamamatsu R11410-21) developed for the XENON1T dark matter search experiment [17]. The new PMTs have lower radioactive 
impurities than the PMTs used in the previous phase of the experiment XENON100, and the collaboration concludes that the new PMTs will not limit the sensitivity of the experiment. The dark matter search by the XENON1T detector will start very soon.

\section{Summary}

Although the evolution of SiPM/MPPSs is very rapid and they have excellent photon detection performances, there are still sufficient reasons to use vacuum PMTs. Development of large aperture PMTs with higher photon detection efficiency is essential for next generation neutrino experiments. Activities for applying innovative technologies which have been established in smaller PMTs to larger aperture PMTs are also progressing (HPD, spherical MCP-PMT, LAPPD, etc.).

\section{References}

[1] Proceedings of this conference (PhotoDet 2015) by B. Lubsandorzhiev

[2] http://www.hyper-k.org/en/index.html

[3] Proceedings of this conference (PhotoDet 2015) by Y. Okajima et al.

[4] Proceedings of this conference (PhotoDet 2015) by D. Fukuda et al.

[5] Proceedings of this conference (PhotoDet 2015) by M. Jiang et al.

[6] Proceedings of this conference (PhotoDet 2015) by Y. Nishimura et al.

[7] Presentation by R. Svoboda at the 6th open meeting for the Hyper-Kamiokande Project.

[8] Presentation by S. Qian at the NDIP2014 conference.

[9] Presentation by P. Coyle at the 3rd Hyper-Kamiokande EU meeting.

[10] G. Barbarino et al., Astropart. Phys. 67 (2015) 18-25.

[11] http://psec.uchicago.edu/

[12] Presentation by K. Byrum at the 13th Pisa Meeting on Advance Detectors.

[13] Proceedings of this conference (PhotoDet 2015) by K. Matsuoka et al.

[14] Presentation by S. Nishida at the NDIP2014 conference.

[15] http://www-sk.icrr.u-tokyo.ac.jp/xmass/index-e.html

[16] Presentation by M. Yamashita at the CIPANP 2015 conference.

[17] E. Aprile et al., Eur. Phys. J. C 75 (2015) no.11, 546. 